\title{
ARCHITECTURE SOLUTIONS FOR INTEGRATING CAD, CAM AND MACHINING IN SMALL COMPANIES
}

\author{
R. Boissier, R. Ponsonnet, M. Raddadi, D. Razafindramary \\ Groupe de Recherche sur la Production Intégrée (GRPI) \\ IUT de St Denis, Université Paris 13. \\ F-93206 St DENIS CEDEX 1, France. \\ Fax.: +33-1-49 4061 96. E-mail :rboissier@d.univ-paris13.fr
}

\begin{abstract}
.
The functions and flows in the process of machining complex geometry part are considered. The computer solutions as well as some of their effective implementation in a demonstration platform are described. The overall efficiency greatly relies on communicative numerical controllers and availability of local and wide area networking facilities.
\end{abstract}

\section{Keywords.}

$\mathrm{CAD}, \mathrm{CAM}, \mathrm{CIM}, \mathrm{CNC}$, quality control, industrial network.

\section{THE NEED FOR NEW PERFORMANCES IN SMALL CNC SPECIALISED COMPANIES}

In the field of mechanical industries, large industrial groups (automotive, aerospace,...) urge their subcontracting partners to adopt $\mathrm{CAD} / \mathrm{CAM}$ tools and quality control procedures to reach the shortest development times. This means difficult choices for small subcontracting companies.

For several years GRPI has been devoting efforts to the design and implementation of performance inductive techniques for a particular category of subcontracting companies : those specialised in the machining of complex geometry parts. A part is said to have a complex 
geometry whenever the cutting path cannot reduce to a mere set of macroscopic linear or circular moves. In which case NC (Numerically Controlled) machining must compulsorily be associated to CAM (Computer Aided Manufacturing) tools. In a few years, NC machining of complex parts has been taking over traditional hand modelling as a result of the spreading of $\mathrm{CAD} / \mathrm{CAM}$ techniques. It concerns :

- many consumer products the shape of which is mainly governed by aesthetics considerations,

- shapes resulting from mathematical optimisation criteria such as from the Finite Element Methods.

Complex shaped parts are seldom machined in large batches because it is a relatively slow and very expensive process. On the other hand this technique is very appropriate in the following cases :

- part prototyping (in the course of the product design phase)

- manufacturing of tools such as moulds, electrodes for electro-erosion, etc.

After considering the main technical activities to be performed in a CNC plant, the solutions studied and implemented will be presented.. The various achievements deal with the preparation, machining and inspection processes, as well as the integration of these processes.

\section{TECHNICAL FUNCTIONS ANALYSIS}

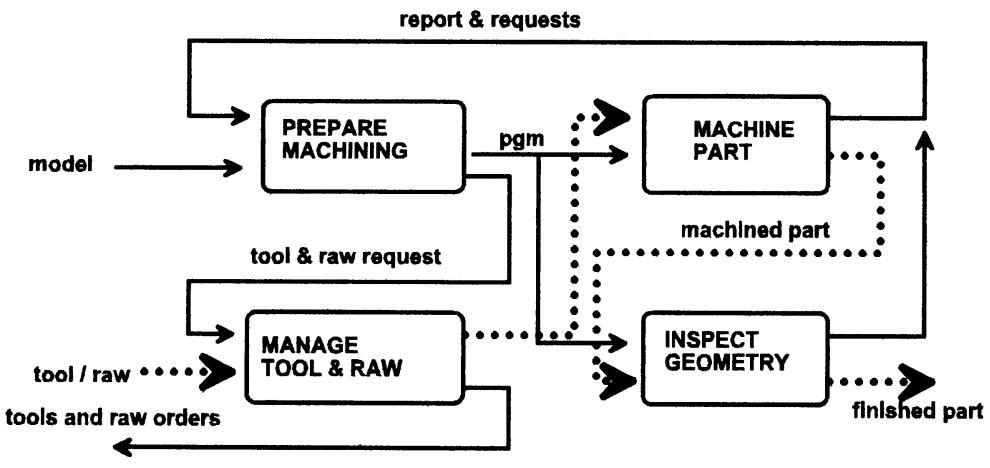

Figure 1 Technical functions in CNC plant, level 1. 
Figure 1 is a SADT-like, level 1, display of the interconnection of the main technical functions to be performed in a typical subcontracting CNC company.

\subsection{Function PREPARE MANUFACTURING can be split into :}

- project file reception : transfer, unpack, store

- pre-analysis of received CAD data : choose and request necessary tools and machines

- geometry analysis and tool path generation

- choice of machining parameters

- machining simulation for error tracking

- post-processing of tool path data into specific machine-tool programs

- selecting elements and surfaces to be inspected

- generate surface sensing sequences

\subsection{Function PERFORM MACHINING receives a PART FILE which consists of :}

- NC machining programs, specific to the considered machine (Tape Files). The operator may be led to chain together several sub-programs, for example several raw and finishing cuts.

- Operator directives : the program or the appended directives may trigger tool search, either in the machine local store (automated or not) or in the workshop central tool store.

The machining data for a part consist of several computer files. The management of this Part File is performed at the machining station by the operator. The problems to be solved at this level are related with the fact that complex shape machining programs consist of an extensive number of very short linearly interpolated moves : most of the Numerical Controllers, except for very recent ones, are unable to memorise data amounting to more than 1 megabyte. On the other hand their computing power is limited to a few tens interpolations per second where a few hundred segments per second would be more appropriate, particularly for making prototypes out of soft polymer material.

\subsection{Function INSPECT PART ultimately warrants conformance of the finished product:}

The Coordinate Measuring station gets a Part Inspection File which consists of : (i) sensing sequences for the surfaces and elements to be inspected, (ii) tolerance margins in the form of associated software gauges. According to the sensing directives, the Coordinate Measuring Machine (MMT) senses the part, determines the part own referential and registers the coordinates of the specified points. These data are then collected and processed in order to 
show the operator whether the part is within the tolerances or not. More accurate and visual interpretation of the results can be performed on the preparation site and decision can then be taken about a bad part to modify any of the CAD/CAM processes.

\subsection{Function MANAGE TOOLS AND MATTER :}

This function is invoked right at the start by the project leader to specify the needs and trigger orders for raw material, equipment, tool preparation or just tools and machine-tool reservation. The Tool Data Base (TDB) is updated by the tool operator in the course of his tool management activity. The TDB is relatively complex because tools are compound objects whose characteristics are time changing due to wear, re-sharpening, re-assembly. The TDB must also manage tool location within the workshop and their allocability to the machines. The data in the TDB should be accessible from any work station. Also the NC machine-tool controllers may memorise tool compensation tables which must be closely linked with the TDB.

\subsection{Needs for COMMUNICATION :}

Links in Figure 1 emphasise the necessity of communication between the previously mentioned functions. In view of relieving the operators from cumbersome administration tasks and avoiding errors meanwhile any information transmission or transcription, the company must be equipped with :

- means for easy local and remote data transfer,

- software interfaces for inter-software communication

- man-machine interfaces for the management of data exchanges and retrieval.

\section{IMPLEMENTATION OF A LIGHT INTEGRATED CNC PLANT.}

In the following, the guiding principle for the choices of architecture has been one of standardisation and simplicity. This principle is well adapted to the limited means of a small company, furthermore it does not block the plant in a one time configuration (Figure 2).

\subsection{Communication.}

\subsubsection{Remote communication.}


Traditional means of communication in which humans interact at both ends remain of utmost importance and new technologies like telefax or electronic mail increase their efficiency.

A national or international automated system for information diffusion or retrieval is also a important factor of productivity. In that respect the French intensively use the TELETEL videotex service which offers :

- access to professional directories, particularly useful when subcontracting is intended;

- access to technical data bases like CETIM's (Centre d'Etude des Industries Mécaniques);

- easy server set up, very useful for a company with many clients who wish to automate information diffusion and order taking.

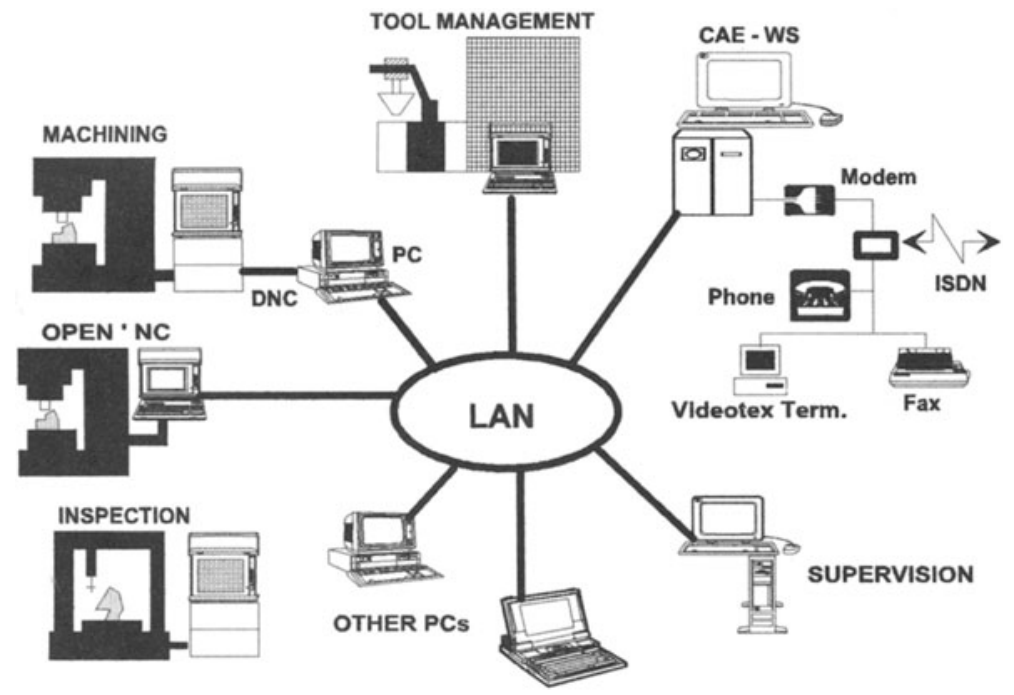

Figure 2 Schematic overview of CNC plant

Beside interactive exchanges, the company needs Digital Data Exchange with their customers for the transfer of manufacturing files and reports, and various solutions can be considered. Due to the variety and evolution of CAD systems and neutral format standards, it may be safer to call for the services of $\mathrm{CAD}$ file transfer nodes were expertise in format translation is concentrated.

As to the physical link, the Integrated Service Data Network (ISDN), in service in many countries, presently seems at short term the best compromise between cost and throughput. ISDN can forward Pulse Code Modulated voice and digital data at the rate of $(2 \times 64+$ 16)Kbits/s. Transmission time for 1 megabyte is then reduced to some $2 \mathrm{mn} / \mathrm{Mbyte}$ which is an improvement by a factor of ten over usual asynchronous Modems. It seems judicious to 
connect at least the technical office to ISDN, one ISDN socket is sufficient to serve all telecommunication usage up to 8 various terminals.

\subsubsection{Local communication.}

Any workstation in the workshop in the plant consists in, integrates or is under control of a standard computer. These computers are CAD/CAM/CAE UNIX workstations, X-Windows terminals and industrial PC/PS microcomputers under DOS or OS/2.

Inter-computer communication is through a standard office Local Area Network such as Ethernet or Token Ring since traffic is intensive only during short bursts at file transfer time. Actual transmission time is some $10 \mathrm{~s} / \mathrm{Mbyte}$ due to protocol overhead and PC's limited throughput). Due to plant heterogeneity, TCP-IP has been adopted as the base protocol for the exchanges. TCP-IP has the advantage of being part of the UNIX/AIX operating system and available on MS-DOS or MS-WINDOWS systems.

\subsection{Preparation of the machining process}

Machining preparation is performed on IBM AIX-RS6000 workstations with high performance graphic controllers. The CAD package is CATIA from Dassault Systems, a surface and solid modeler. CATIA integrates CAM modules for multiaxis lathes and milling centres. The $\mathrm{CAD} / \mathrm{CAM}$ operator gets delivery of the remote geometric model of the part to be machined and defines on which surface elements and along which curves the tool end will be moving, actually he must be a specialist in both CAD and CAM. The CATIA CAM module allows for introducing the surface tolerance and tool geometry, and simulating tool path. This visual check is a protection against the most obvious errors.

Machining data are produced in an intermediate APT-like format. These data must then be adapted to the selected machine-tool characteristics through a so called Post-Processor interface which produces machine specific Tape Files. Modular post-processors for multiaxis machining centres have been developed. The ISO Tape Files pertaining to a given part are grouped in a Part File and become available on request from the machining station operator.

The CAD/CAM workstations also support a Computer Aided Inspection software, VALISYS, which is interfaced with the CATIA generated models. On the graphic display, the $\mathrm{CAD} / \mathrm{CAM}$ operator defines the tolerancing type with graphic ISO/ANSI symbols which are translated into Virtual Gauges. For any component surface to be inspected, a grid of test points is generated. Both inspection sequence and tolerancing data are available from the Inspection Station through the local network. Conversely the CAD/CAM operator can retrieve data issued during an inspection session and visually compare the specified and measured surfaces. 


\subsection{Machining stations}

\subsubsection{Computer as a Data Store and a Machine-tool Supervisor.}

At the beginning of this project development, one urging need was present : how to carry out complex prototypes machining on a modern milling centre, yet equipped with a poor $60 \mathrm{Kbytes}$ program memory. The first answer was the use of a Direct Numerical Control (DNC) mode in which the PC computer standing by the machine-tool manages data transfer along a $9600 \mathrm{bits} / \mathrm{s}$ asynchronous line according to a safe block mode protocol.. This was tested by executing machining programs over 1 megabyte long. Extension to PC control of several machines, using an intelligent communication card was also considered. When later on CAD/CAM workstations and a LAN were installed on the site, it was easy to include calls to TCP-IP application commands in the DNC software so as to let the PC-DNC operator ask for the downloading of a Part File from any CAD/CAM workstation (Boissier, 1988), (Razafindramary, 1991).

\subsubsection{PC Computer as the kernel of an Open Numerical Controller.}

The preceding tests made clear a serious limitation of the microprocessor based CNC controllers in the 80 's : a low interpolation speed (5-10 blocks/s), affordable for macroscopic moves but speed limiting on short segment surface following.

Searching at once for high interpolation speed, communicativity, evolutivity and low cost, we developed a concept of OPEN NC using a standard PC computer component for managing dialogue with the operator, and the workshop, and a specialised module for rapid interpolation.

The machine functions and dynamics have been specified in Figure 3, using the Ward \& Mellor hierarchical formalism(Ward, 1986). This specification has been mapped into the architecture in Figure 4 (Raddadi, 1991,1993).

The OPEN NC is composed of an industrial PC working as operator interface, program store with mass memory and workshop communication unit with a LAN adapter and the TCP-IP protocol. Machine-tool axes control (3 axes, extensible to 5) is performed by an add-on card from the Delta Tau Company whose specialised Digital Signal Processor (Motorola DSP 56000 ) achieves in a very small volume interesting interpolation performances. 


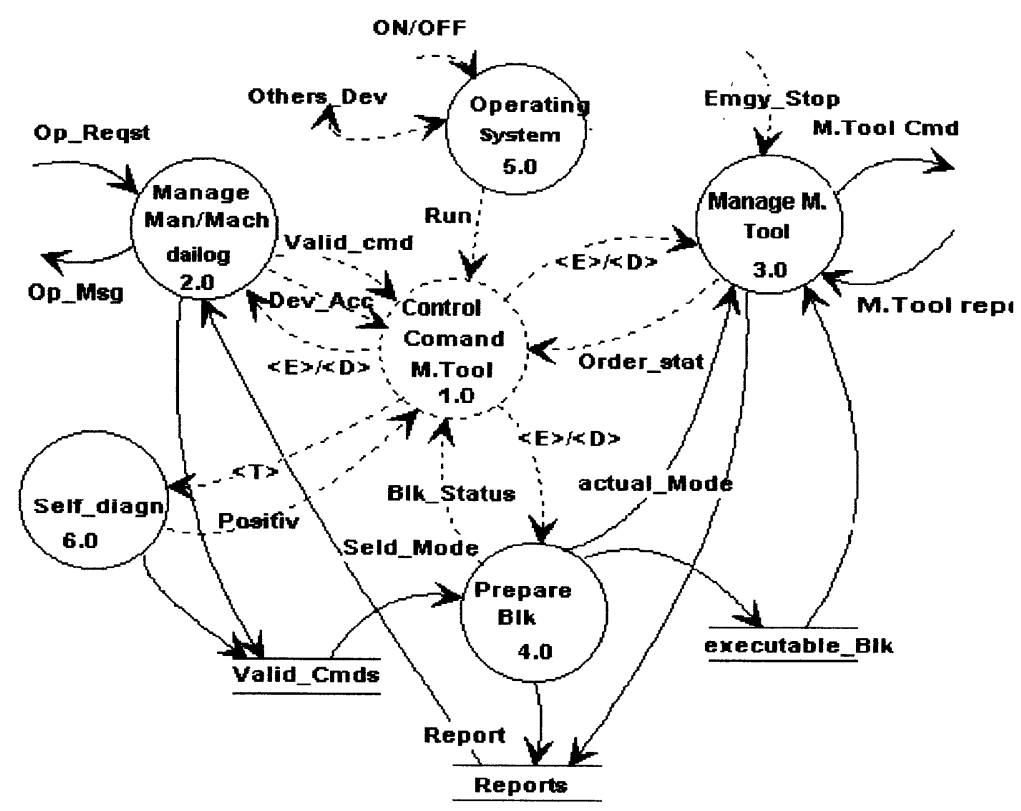

Figure 3 Functional and dynamic specification of OPEN NC

Control of a machine tool and its environment implies some logic and sequential functions, these are specified with Sequential Function Charts or Grafcet (IEC, 1992) and implemented as cyclic I/O tasks within the Real Time IBM-ACS environment (IBM, 1993). I/O data are conveyed to the operative part through a $1 \mathrm{Mbit} / \mathrm{s}$ field bus, such a link to remote $\mathrm{I} / \mathrm{O}$ reduces cabling time and errors.

The achieved OPEN NC is very compact, very modular and extensible, it allows easy transformation of a mechanically sound machine tool with obsolete control into a very performing and evolutive industrial system.

The IBM-France Company have seen, in our solution, the possibility to directly integrate machining stations into their overall industrial communication architecture (IBM, 1993). Actually the control architecture is adaptable to a great variety of contexts.

The need and interest for openness in a machine-tool controller is real and has given way to several studies and realisations. A critic of traditional controllers has been presented in (Grégoire, 1989) together with the design of an experimental modular system, while recently NC manufacturer NUM SA expressed their interest for such systems and announced an architecture somewhat different than ours, in which a PC-OS/2 compatible card is plugged into 
the VME bus based existing numerical controller, allowing the local installation of DOS or OS/2 applications (Pritschow, 1993).

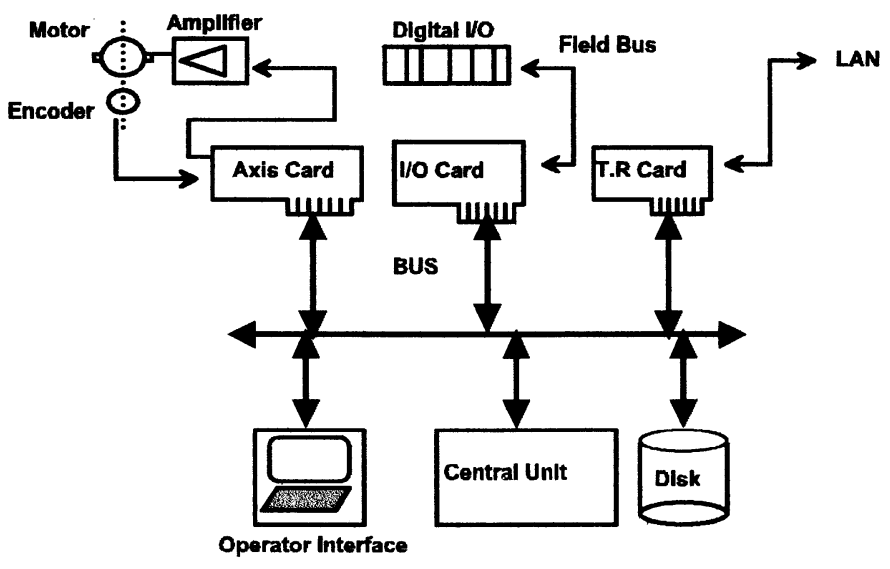

Figure 4 Architecture of OPEN NC

\subsection{Coordinate Inspection Station}

Integration of a coordinate inspection stand in the plant profits from the above mentioned developments. The VALISYS modules needed at inspection stand are installed in an AIX operated IBM-PS/2, just by the DEA Coordinate Measuring Machine (CMM). This microcomputer serves as a relay between the technical office and the CMM, it translates the VALISYS sensing instructions into specific DEA instructions which are downloaded into the CMM controller through an asynchronous line. Conversely, coordinate data retrieved from the CMM are locally formatted for the purpose of VALISYS processing.

\section{CONCLUSION.}

In this paper some particular aspects of CIM have been highlighted. We wish to make clear, particularly to industrials that in small plants integration needs a relatively modest investment compared to machine-tools ones. The light communication system, simple gateways and versatile Open Numerical Control we have set-up should contribute to CNC plant performance and leave more time for the operators to practise their job skills. Work in progress includes a methodological object oriented approach to tackle overall manufacturing management, particularly tool management, with inclusion of MMS primitives (Lefebvre, 1994). 


\section{ACKNOWLEDGEMENTS}

The authors wish to thank Mr MOURIER, IBM consulting engineer, and his colleagues as well as Mr MONTES, IUT workshop manager, and the IUT technicians for their fruitful help.

\section{REFERENCES}

Boissier R. et Al. (1988).La chaîne CAO-Usinage dans un îlot de fabrication de pièces à géomètrie complexe. Rev. Aut. \& Prod. Appl. Paris. 1(4), 38 - 49

Grégoire J-Ch (1989). Architecture de commandes numériques des machines-outils. PhD Thesis, EPFL-Lausanne.

IBM (1993). Dossiers de l'Informatique Industrielle. 4411-01/93. IBM Paris.

IEC (1992). Sequential Function Charts. International IEC-848 standard. IEC,Geneva.

Lefebvre M. (1994). MMS sur TCP-IP, une solution qui utilise l'appel de procédure à distance. D. Ing. dissertation., CNAM-Paris.

Pritschow G. (1993).Il faut ouvrir les CN. NUM Information (18),13. Argenteuil.

Raddadi (1992). Elaboration d'un DCN multiaxes ouvert. PhD Thesis, Université de Nancy 1.

Raddadi M. et Al. (1993).Spécification temps-réel et réalisation d'une commande numérique ouverte. Rev. Aut. \& Prod. Appl. 6(3), 303 - 322. Paris.

Razafindramary D. (1991). Conduite de Systèmes de Fabrication. PhD Thesis, Université de Paris-Nord.

Ward S. and Mellor (1986). Structured development of Real Time Systems. Yourdon Press, Englewood Cliffs.

\section{BIOGRAPHY}

Raymond Boissier graduated from Ecole Supérieure d'Electricité and is a Doctor in Physics, he has specialised in industrial automation and computing. Raymond Ponsonnet graduated from Ecole Normale Supérieure de Cachan and is a Doctor in Science He is the head of GRPI and has specialised in robotics and complex surface inspection. Mohammed Raddadi graduated from Centre d'Etudes Supérieures des Techniques Industrielles and is a Doctor from Université de Nancy 1 . He has specialised in machine-tool control.Donné Razafindramary got his Engineering Diploma in telecommunication and his Doctorate from Université Paris-Nord. He has specialised in industrial computing and workshop communication. 